\title{
Investigation on nutrition status and clinical outcome of patients with common cancers in Chinese patients: a multicenter prospective study protocol
}

\begin{abstract}
Hongxia Xu' ${ }^{1}$, Chunhua Song ${ }^{2}$, Chang Wang ${ }^{3}$, Zhenming Fu' ${ }^{4}$, Zengqing Guo ${ }^{5}$, Yuan Lin ${ }^{6}$, Yingying Shi ${ }^{7}$, Wen $\mathrm{Hu}^{8}$, Yi Ba ${ }^{9}$, Suyi $\mathrm{Li}^{10}$, Zengning $\mathrm{Li}^{11}$, Kunhua Wang ${ }^{12}$, Jing Wu ${ }^{13}$, Ying $\mathrm{He}^{14}$, Jiajun Yang ${ }^{15}$, Conghua Xie ${ }^{16}$, Fuxiang Zhou ${ }^{16}$, Xinxia Song ${ }^{17}$, Gongyan Chen ${ }^{18}$, Wenjun Ma $^{19}$, Suxia Luo ${ }^{20}$, Zihua Chen ${ }^{21}$, Minghua Cong ${ }^{22}$, $\mathrm{Hu} \mathrm{Ma}^{23}$, Chunling Zhou ${ }^{24}$, Wei Wang ${ }^{25}$, Qi Luo ${ }^{26}$, Yongmei Shi $^{27}$, Yumei Qi ${ }^{28}$, Haiping Jiang ${ }^{29}$, Wenxian Guan ${ }^{30}$, Junqiang Chen ${ }^{31}$, Jiaxin Chen ${ }^{32}$, Yu Fang ${ }^{33}$, Lan Zhou ${ }^{34}$, Yongdong Feng ${ }^{35}$, Rongshao Tan ${ }^{36}$, Tao Li $^{37}$, Junwen Ou ${ }^{38}$, Qingchuan $\mathrm{Zhao}^{39}$, Jianxiong $\mathrm{Wu}^{40}$, Min Weng ${ }^{41}$, Qinghua $\mathrm{Yao}^{42}$, Wei $\mathrm{Li}^{3}$, Hanping Shi ${ }^{43,44 *}$
\end{abstract}

\begin{abstract}
${ }^{1}$ Department of Clinical Nutrition, Daping Hospital, Army Medical University, Chongqing, China, ${ }^{2}$ Department of Epidemiology, College of Public Health, Zhengzhou University, Zhengzhou, Henan, China, ${ }^{3}$ Cancer Center, The First Hospital of Jilin University, Changchun, Jilin, China, ${ }^{4}$ Cancer Center, Renmin Hospital of Wuhan University, Wuhan, Hubei, China, ${ }^{5}$ Department of Medical Oncology, Fujian Cancer Hospital, Fujian Medical University Cancer Hospital, Fuzhou, Fujian, China, ${ }^{6}$ Department of Gastrointestinal Surgery, Affiliated Cancer Hospital of Guangxi Medical University, Nanning, Guangxi, China, ${ }^{7}$ Department of Surgery, The First Affiliated Hospital of SunYat-sen University, Guangzhou, Guangdong, China, ${ }^{8}$ Department of Clinical Nutrition, West China Hospital of Sichuan University, Chengdu, Sichuan, China, ${ }^{9}$ Department of Gastrointestinal Oncology, Tianjin Medical University Cancer Institute and Hospital, National Clinical Research Center for Cancer, Tianjin Key Laboratory of Cancer Prevention and Therapy, Tianjin, China, ${ }^{10}$ Department of Nutrition and Metabolism of Oncology, Affiliated Provincial Hospital of Anhui Medical University, Hefei, Anhui, China, ${ }^{11}$ Department of Clinical Nutrition, The First Hospital of Hebei Medical University, Shijiazhuang, Hebei, China, ${ }^{12}$ Department of Gastrointestinal Surgery, Institute of Gastroenterology, The First Affiliated Hospital of Kunming Medical University, Kunming, Yunnan, China, ${ }^{13}$ Department of Clinical Nutrition, The First People's Hospital of Kashi, Xinjiang, China, ${ }^{14}$ Department of Clinical Nutrition, Chongqing General Hospital, Chongqing, China, ${ }^{15}$ Department of Colorectal and Anal Surgery, Huizhou Municipal Central Hospital, Huizhou, Guangdong, China, ${ }^{16}$ Department of Radiation and Medical Oncology, Zhongnan Hospital of Wuhan University, Wuhan, Hubei, China, ${ }^{17}$ Department of Oncology, Xingtai People's Hospital, Hebei Medical University, Xingtai, Hebei, China, ${ }^{18}$ Harbin Medical University Cancer Hospital, Harbin, Heilongjiang, China, ${ }^{19}$ Department of Nutrition, Guangdong General Hospital, Guangdong Academy of Medical Sciences, Guangzhou, Guangdong, China, ${ }^{20}$ Department of Oncology, Affiliated Cancer Hospital of Zhengzhou University and Henan Cancer Hospital, Zhengzhou, Henan, China, ${ }^{21}$ Department of General Surgery, Xiangya Hospital, Central South University, Changsha, Hunan, China, ${ }^{22}$ Comprehensive Oncology Department, National Cancer Center or Cancer Hospital, Chinese Academy of Medical Sciences and Peking Union Medical College, Beijing, China; ${ }^{23}$ Department of Oncology, Affiliated Hospital of Zunyi Medical University, Zunyi, Guizhou, China, ${ }^{24}$ The Fourth Affiliated Hospital, Harbin Medical University, Harbin, Heilongjiang, China, ${ }^{25}$ Cancer center, The First People's Hospital of Foshan, Foshan, Guangdong, China, ${ }^{26}$ Department of Gastrointestinal Tumor Surgery, The First Affiliated Hospital, Xiamen University, Xiamen, Fujian, China, ${ }^{27}$ Department of Nutrition, Ruijin Hospital, Shanghai Jiao Tong University School of Medicine, Shanghai, China, ${ }^{28}$ Department of Nutrition, Tianjin Third Central Hospital, Tianjin, China, ${ }^{29}$ Department of Surgery, The First Affiliated Hospital of Jinan University, Guangzhou, Guangdong, China, ${ }^{30}$ Department of General Surgery, Nanjing Drum Tower Hospital, The Affiliated Hospital of Nanjing University Medical School, Nanjing, Jiangsu, China, ${ }^{31}$ Department of Gastrointestinal Surgery, First Affiliated Hospital of Guangxi Medical University, Nanning, Guangxi, China, ${ }^{32}$ Department of Radiation and Medical Oncology, People's Hospital of Guangxi Zhuang Autonomous Region, Nanning, Guangxi, China, ${ }^{33}$ Department of Clinical Nutrition, Peking University Cancer Hospital and Institute, Beijing, China, ${ }^{34}$ Department of Nutrition, Third Affiliated Hospital of Kunming Medical College, Tumor Hospital of Yunnan Province, Kunming, Yunnan, China, ${ }^{35}$ Department of Surgery, Tongji Hospital, Tongji Medical College, Huazhong University of Science and Technology, Wuhan, China, ${ }^{36}$ Department of Nutrition, Guangzhou Red Cross Hospital, Guangzhou, Guangdong, China, ${ }^{37}$ Department of Radiotherapy, Sichuan Cancer Hospital \& Institute, Sichuan Cancer Center, School of Medicine, University of Electronic Science and Technology of China Chengdu, Sichuan, China, ${ }^{38}$ Department of Clinical Nutrition, Clifford Hospital, Guangzhou University of Chinese Medicine, Guangzhou, Guangdong, China, ${ }^{39}$ Department of Digestive Diseases, Xijing Hospital, Fourth Military Medical University, Xi'an, Shanxi, China, ${ }^{40}$ Department of Hepatobiliary Surgery, National Cancer Center or Cancer Hospital, Chinese Academy of Medical Sciences and Peking Union Medical College, Beijing, China,
\end{abstract}




\begin{abstract}
${ }^{41}$ Department of Clinical Nutrition, The First Affiliated Hospital of Kunming Medical University, Kunming, Yunnan, China, ${ }^{42}$ Department of Integrated Traditional Chinese and Western Medicine, Zhejiang Cancer Hospital and Key Laboratory of Traditional Chinese Medicine Oncology, Zhejiang Cancer Hospital, Hangzhou, Zhejiang, China, ${ }^{43}$ Department of Gastrointestinal Surgery, Department of Clinical Nutrition, Beijing Shijitan Hospital, Beijing, China, ${ }^{44}$ Department of Oncology, Capital Medical University or Ninth Clinical Medical College, Peking University, Beijing, China
\end{abstract}

Received: 02 February 2020

Revised: 17 February 2020

Accepted: 20 February 2020

\title{
*Correspondence: \\ Dr. Hanping Shi, \\ E-mail: shihp@vip.163.com
}

Copyright: $\odot$ the author(s), publisher and licensee Medip Academy. This is an open-access article distributed under the terms of the Creative Commons Attribution Non-Commercial License, which permits unrestricted non-commercial use, distribution, and reproduction in any medium, provided the original work is properly cited.

\section{ABSTRACT}

Background: Malnutrition is common in patients with cancer, which adversely affects the survival and quality of life of cancer patients. However, there is no national data on the prevalence of malnutrition in Chinese cancer patients. This study aims to evaluate the prevalence of malnutrition and quality of life (QOL) of Chinese patients with local regional, recurrent or metastatic cancer, to address the prognostic value of nutritional status and QOL on the survival of cancer patients in China and to validate the patient-generated subjective global assessment (PG-SGA) questionnaire in Chinese cancer patients.

Methods: This is an observational, multi-centered, and hospital-based prospective cohort study. We aimed to recruit 50,000 cancer patients (age 18 and above) over an 8-year period. Data collection will occur within $48 \mathrm{hr}$ after patients are admitted to hospital, 30-days after hospital admission, and the follow-up will be conducted 1-8 years after enrolment. The primary outcome is overall survival, and secondary outcomes are length of hospital stay and hospital costs. Factors measured are demographic characteristics, tumor characteristics, anthropometry measurements, hematological measurement, body composition, PG-SGA scores, Karnofsky performance status scores, and QLQ C30 scores. This protocol was approved by local ethical committees of all the participant hospitals.

Conclusions: This multi-centered, large-scale, long-time follow-up prospective study will help diagnose malnutrition in cancer patients in China, and identify the related risk factors associated with the negative outcomes. The anticipated results will highlight the need for a truly scientific appraisal of nutrition therapy, and help to improve outcomes among cancer patients in China.

Trial Registration: The trial has been registered with the Chinese Clinical Trial Registry, ChiCTR1800020329. Registered on 19 December 2018.

Keywords: Nutritional status, Nutritional assessment, Malnutrition, Patient-generated subjective global assessment, Bodyweight loss, Karnofsky performance status score, Quality of life

\section{INTRODUCTION}

The prevalence of cancer has been increasing in the last decades all over the world, including in China. ${ }^{1,2}$ The number of cancer survivors continues to increase owing to the advances in early detection and cancer treatment, and growth of the population. ${ }^{3}$ In China, cancer mortality has been increasing, making cancer the leading cause of death since $2010 .{ }^{2}$ The mortality of cancer patients in China is remarkably higher than that in other continents, especially in Europe and in North America. ${ }^{2}$ In addition, the incidence of cancer has been increasing at an alarming rate in China, posing a major public health burden. $^{2}$
It is increasingly accepted that the nutritional status affects the therapeutic outcome and quality of life of cancer patients..$^{4-7}$ Malnutrition is common in patients with cance. ${ }^{4-7}$ Cancer itself and cancer treatment decrease the energy and nutrients intake and cause metabolic alterations, resulting in malnutrition in cancer patients. ${ }^{8-17}$ The prevalence of malnutrition is reported to be from about $20 \%$ to more than $70 \%$ in worldwide studies; the differences may be related to age, cancer type, cancer stage, region and social economic status. ${ }^{4-7,11,12}$ The proportion of patients with weight loss at diagnosis often ranges from $15 \%$ to $40 \%,{ }^{5}$ and may increase up to $85 \%$ in patients with certain cancers (e.g., pancreatic cancer). ${ }^{6}$ For example, a large nationwide Brazilian study started in 
1996, which revealed a global rate of $48.1 \%$ (by subjective global assessment (SGA)) malnourished subjects (12.6\% severe). ${ }^{13}$ Additionally, the incidence of malnutrition increases as the disease progresses and may affect up to $80 \%$ of patients. $., 7,11,14,15$

Malnutrition adversely affects survival and quality of life in cancer patients. ${ }^{16}$ Several studies have shown that malnutrition is an independent risk factor for more complications and increased length of hospital stay and costs, and mortality. ${ }^{17,18}$ It is estimated that the deaths of $10-20 \%$ of patients with cancer can be attributed to malnutrition rather than to the malignancy itself, including patients with gynecological cancer or gastrointestinal cancer. ${ }^{19,21,22}$ Moreover, cancer treatments are hampered by the development of malnutrition in many cancer patients. 5,23

China has the $20 \%$ cancer cases and the $25 \%$ of the deaths for all the cancer worldwide. ${ }^{1}$ Many of the cancer deaths can be prevented through risk factor modification and improving the effectiveness of clinical care, including nutritional intervention in cancer patients with malnutrition..$^{9,24-28}$ Recently the nutrition status of cancer patients has caught the attention of Chinese surgeons, physicians and dieticians. ${ }^{22,29-35}$ It is reported that the prevalence of malnutrition ranged from $12.6 \%$ to $54 \%$, depending on the cancer type, cancer stage, and patient age. ${ }^{22,30,32,33,35}$ The benefits of nutritional intervention have been shown in cancer patients with malnutrition. ${ }^{8,25,27}$ However, the sample size in the reported studies is relatively small, and most of them focus on the gastrointestinal cancer and lung cancer, not including many other frequently diagnosed cancers among men and women. Although the consequences of malnutrition of cancer patients are well known and nutritional support is widely recommended for the malnutritional patients in Western countries, the prevalence of malnutrition in Chinese cancer patients remains largely unknown. Therefore, there is a need urgent to investigate the nutritional status of cancer patients in different regions of China, to facilitate the development of effective approaches for preventing and treating malnutrition with an aim to improve the clinical outcomes and quality of life among cancer patients.

In 2013, Chinese Society of Nutritional Oncology (CSNO) initiated a clinical research project, the Investigation on Nutrition Status and Its Clinical Outcome of Common Cancers (INSCOC). More than 80 hospitals all over the China have participated in this project since December 2013. The aim of this study was to determine the prevalence of malnutrition in inpatients cancer patients in China and its relationship to physical performance and quality of life. This study would provide the evidence supporting the prognostic value of nutritional status on the mortality of common cancers, thus highlight the needs for a truly scientific appraisal of nutrition therapy in clinical care of cancer patients. In the present report, we present the study design and methodology of INSCOC, providing the rationale for future follow up plan to generate a large, prospective cohort study.

\section{Aims and hypotheses}

The primary endpoint is to investigate the prevalence of malnutrition in Chinese patients with local regional, recurrent or metastatic cancer, when they are admitted to hospital, during the hospital stay, and in clinical follow ups. Secondary endpoints include to explore whether nutrition status, physical performance, and quality of life, at admission, can predict the overall survival of common cancer patients; to explore the association between the nutrition status and different demographic characteristics among the studied cancer patients; to assess whether cancer patients with malnutrition receive proper nutritional support during their hospital stay, including enteral nutrition $(\mathrm{EN})$ and parenteral nutrition $(\mathrm{PN})$; to validate the patient generated-subjective global assessment (PG-SGA) questionnaire for Chinese cancer patients and to create a brief Chinese PG-SGA, if necessary; and to develop a predictive model of nutritional index for survival of cancer patients.

\section{METHODS}

\section{Study design}

The design of the study is multi-centered, hospital-based prospective cohort study. Cancer patients with one of eighteen types of common malignant tumors are recruited to the study who are admitted to one of the 80 participating hospitals in China. The 80 hospitals are from 16 provinces of China in different regions. We aim to recruit 50,000 cancer patients over an 8-year period. Data collection will occur at baseline (within 48 hours of hospital admission), the current hospital stays, at 30-days since the first admission, and 1-8 years following recruitment. The minimum number of patients recruited from each hospital is set to be 100, with completed data meeting study requirement and data criteria (Figure 1).

\section{Patient recruitment}

All the patients were recruited by clinical investigators in various departments of the participating hospitals, including departments of medical oncology, hematology, radiation oncology, surgical oncology, palliative care, hepatology, gastroenterology, urology, gynecology, pneumology and neurology.

\section{Eligibility criteria}

Patients diagnosed with one of the following 18 different types of malignant tumors: lung cancer, gastric cancer, liver cancer, colorectal cancer, breast cancer, esophageal cancer, cervical cancer, endometrial cancer, nasopharyngeal carcinoma, malignant lymphoma, leukemia, pancreatic cancer, ovarian cancer, prostate 
cancer, bladder cancer, brain tumor, biliary tract malignant tumors and gastric stromal tumors. Cancer records were supplied with the anatomical site coded of the tenth edition of the International Classification of Disease (ICD-10).

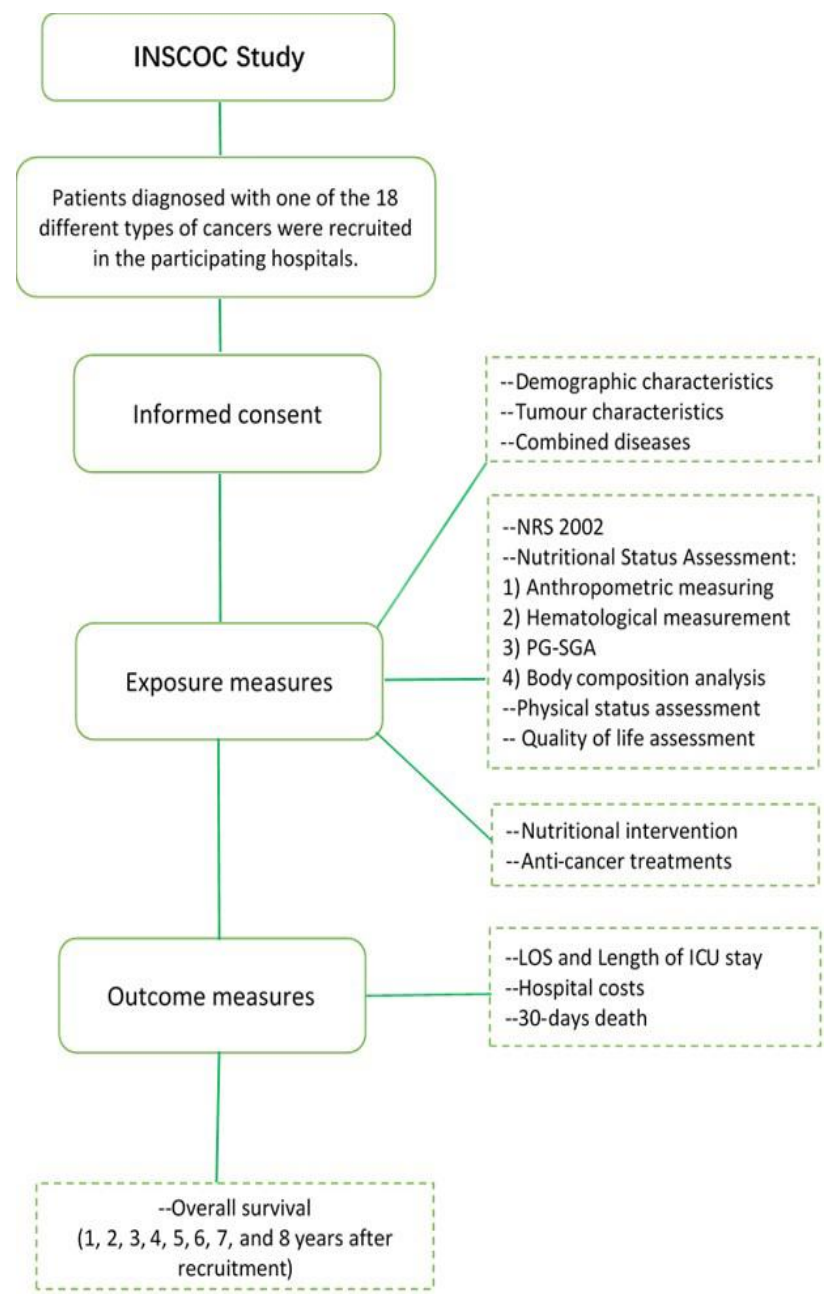

Figure 1: Overview and design of the INSCOC study.

The inclusion criteria are aged 18 and above, hospitalized cancer patient with tumor properly diagnosed and staged as local, metastatic and/or loco-regional relapse, cancer diagnosis confirmed by pathology, conscious, no communication barriers, able to answer the questionnaires of the study, willing to participate in this study and signing an informed consent form. The exclusion criteria are hospitalized more than two times during our investigation, with organ transplantation, pregnant woman, diagnosed with human immuno-deficiency virus (HIV) infection or acquired immune deficiency syndrome (AIDS) and admitted to intensive care unit (ICU) at the beginning of recruitment.

\section{Sample size calculation}

Calculation of the sample size was based on the study of simple random sampling (SRS). According to the data from our pilot study (INSCOC project from 2013), the rate of malnutrition among inpatients with cancer was $26.58 \%$ (PG-SGA $\geq 9$ ), which was the estimate of $\pi$ in this study, $\alpha=0.05, Z_{\alpha / 2}=1.96$.

Absolute maximum permissible error $\delta$ (About $10 \%$ of the incidence $)=p-\pi$.

Computational formula: $N_{S R S}=\mathrm{Z}_{\alpha / 2}^{2} \times \pi \times(1-\pi) / \delta^{2}$ people for each stratum).

Design effect $($ deff $)=2.5$ and the actual requirement for each stratum is 2,630. Total sample size calculated by gender, Central, South, North, South, East and West.

$2630 \times 5$ (Central, South, North, South, East) $\times 2$ (gender) $=26300$.

From December 2013 to December 2020 (7 years), 50,000 inpatients will be recruited into the study.

\section{Ethical considerations}

Written informed consent is required to be obtained from every patient. The study protocol and procedures were first approved by the Ethical Committee of the First Affiliated Hospital of SunYat-sen University on 2nd January, 2013. Then, the study protocol and procedures were and or will be approved by the local ethical committees of each of the participating hospitals.

\section{Measures}

Baseline survey for demographic characteristics and tumour characteristics

All the demographic characteristics which have been indicated to be related to the nutritional status of cancer patients were investigated in this study. The detailed personal information including age, gender, nationality, family history of cancer, residence, medical insurance, education level, occupation, smoking, alcohol drinking, and tea drinking, were collected by professional personnel through face to face interview.

Tumour characteristics, including cancer type, cancer stage (for first diagnosis, and for this admission diagnosis to hospital, pTNM or cTNM cancer stages), tumor differentiation, tumor-burdened state, and anti-cancer treatments before this admission (chemotherapy, radiotherapy, surgery, neoadjuant chemoradiotherapy, targeted therapy, and endocrine therapy) were investigated by professional interviewers.

\section{Combined diseases}

Some combined diseases, which could affect the nutritional status were investigated at the admission to the hospital, including hepatic cirrhosis, chronic hepatitis, chronic pancreatitis, stroke, chronic obstructive pulmonary disease (COPD), myocardial infarction, 
diabetes, hypertension, coronary heart disease, anemia, hyperthyroidism, hypothyroidism, chronic nephrosis, dialysis treatment, osteoporosis, ulcerative colitis, Crohn's disease, chronic diseases of the biliary system, systemic lupus erythematosus and tuberculosis.

\section{Exposure measures}

\section{Nutritional risk screening 2002}

Nutritional risk screening 2002 (NRS 2002) classifies the nutritional risk based on BMI, percentage of recent weight loss, recent change in food intake, and disease severity. One point is added if patients are 70 years or above. When the final score is 3 points, the patient is considered at nutritional risk. ${ }^{36}$ ESPEN expert group and ESPEN guidelines recommend that all patients with cancer be screened for nutritional risk early in the course of their care. ${ }^{12}$

\section{Nutritional status assessment}

\section{Anthropometric measuring}

Anthropometric measurements are performed by trained medical professionals using a standardized protocol. The anthropometric measurement includes height, body weight, body mass index (BMI), arm circumference (AC), arm muscle circumference (AMC), triceps skin fold (TS, nonprofit arm), and calf circumference (CC, left calf). Body weight and height are measured in light indoor clothing without shoes, to the nearest $0.1 \mathrm{~kg}$ and $0.1 \mathrm{~cm}$, respectively.

BMI is then calculated as weight $(\mathrm{kg}) /$ height $(\mathrm{m})^{2}$.

$\mathrm{AMC}$ is then calculated as $\mathrm{AC}(\mathrm{mm})-3.14 \times \mathrm{TSF}(\mathrm{mm})$.

The hand grip strength (HGS, non-dominant hand) is measured by an electronic hand grip dynamometer (CAMRY, Model EH101, Guagndong, China). Patients are asked to stand comfortably, then to perform 3 maximal isometric contractions $30 \mathrm{~s}$ apart using their non-dominant hand.

\section{Hematological measurement}

Admission hematological measurements including red blood cell count (RBC), blood platelet count, total lymphocyte counts, hemoglobin, albumin, prealbumin, total protein, creatinine, C-reaction protein (CRP), IL-1, IL-6 and TNF- $\alpha$ are performed by clinical labs of the participating hospitals.

\section{Patient generated-subjective global assessment}

Patient generated-subjective global assessment (PGSGA) is a nutritional assessment method adapted by Ottery for cancer patients. ${ }^{24}$ It consists of two sections, the first section includes questions about recent weight loss, food intake, symptoms that could interfere with food intake and patients' physical activity. Weight change is recorded beginning at 1 and 6 months before admission, and percent weight change is calculated as (currentprevious weight/previous weight $) \times 100 \%$. Where possible, 1-month weight change is used; otherwise, it is imputed from 6-month weight change, with minimal impact on interpretation of the results.

In the second section, information is collected about disease and metabolic needs while a patient's physical examination is carried out. Each item of PG-SGA rated has a score and the final score indicates if a nutritional intervention is needed. It also originates an overall subjective rating, indicating that a patient may be "well nourished or anabolic (PG-SGA score 0-1)"; "at nutritional risk or moderate under nutrition (PG-SGA score 2-8)"; or "severe under nutrition (PG-SGA score $\geq 9$ )". A score $\geq 9$ indicates a critical need for nutrition intervention.

\section{Body composition analysis}

Body composition analysis reveals loss of muscle mass, and depletion of fat stores in malnourished cancer patients. Body composition assessments are completed by central labs or hospitals. Participants are assessed with a multifrequency bioelectrical impedance (InBody720 or InBodyS10; Biospace, Tokyo, Japan). With this method, body weight, BMI, fat mass, and skeletal muscle mass are automatically and simultaneously measured. Skeletal muscle mass is also expressed as a percentage with respect to a standard skeletal muscle mass values as defined according to the age, sex, and height of each patient.

\section{Physical status assessment}

Cancer patients' physical status assessed by scores of Karnofsky performance status (KPS) questionnaire., ${ }^{9,24}$ Scores 80-100 indicate that the patients be able to carry on normal activity and to work, no special care is needed; scores 50-70 indicate that patients be unable to work, but able to live at home and care for most personal needs, varying amount of assistance is needed; scores 0-40 indicated that patients be unable to care for self, requires equivalent of institutional or hospital care, and his/her disease may be progressing rapidly; and the score 0 indicates that the patient is dead.

\section{Quality of life assessment}

The quality of life for all the participating patients is measured using the European Organization for Research and Treatment of cancer (EORTC) QLQ-C30 Version $3 .^{37}$ This patient-based instrument comprises of 30 items and five functional scales (physical, role, cognitive, emotional and social), three symptom scales (fatigue, pain and nausea/vomiting), global health status and QoL scales. QLQ-C30 results are linearly converted to a score 
out of 100, with higher score reflecting a higher QoL. The QoL scores are calculated according to the scoring manual. ${ }^{38}$

\section{Nutritional intervention}

The investigators follow the patients and record whether they receive parenteral nutrition $(\mathrm{PN})$ or enteral nutrition (EN) intervention. $\mathrm{PN}$ is defined as nutrients administered intravenously and consisting of a combination of amino acids, carbohydrate, and fat, or any amino acids in fusion, or any lipid emulsion infusion. EN is defined as oral nutrient supplementation and tube feeding that provides at least $10 \mathrm{kcal} / \mathrm{kg} / \mathrm{d}$, according to the guideline from European Society of Parenteral and Enteral Nutrition. ${ }^{39}$ Overall, a minimum of $3 \mathrm{~d}$ of PN or EN is considered nutritional support. ${ }^{40}$ Some cancer patients may receive EN plus PN.

\section{Anti-cancer treatments}

All the anti-cancer treatments are prospectively investigated by professional interviewers during the hospital stay, including chemotherapy, radiotherapy, surgery, neoadjuant chemoradiotherapy, targeted therapy and endocrine therapy.

\section{Outcome measures}

\section{Length of hospital stay and length of ICU stay}

Length of hospital stay is defined as the duration between the admission and the discharge or death. Some of patients may be admitted into the ICU during this hospital stay. Length of ICU stay is defined as the duration between the admission of ICU and discharge from ICU or death.

\section{Hospital costs}

Hospital costs are obtained from patients' medical record for this admission. Total hospital costs for this admission and the daily hospital infirmary rate are used. All the costs of this admission in the medical record are included, such as operation, drugs, tests, materials, bed costs, nursing, nutritional intervention and physical therapy, among other costs.

\section{0-days death}

The death in the 30 days within this hospitalization is obtained from medical record or telephone follow-up interview after hospital discharge, which is taken as an assessment of short-term clinical outcome in this study.

\section{Overall survival}

All the enrolled patients with be followed-up annually for 8 consecutive years. The death information will be collected by telephone follow-up interview and recorded at $1,2,3,4,5,6,7$, and 8 years after recruitment. The data are used to calculate the long-term survival rate in the study.

\section{Data collection}

At the time of recruitment to the study, the intervieweradministered questionnaire consists of the following baseline information: 1) demographic characteristics, 2) tumor characteristics, 3) combines diseases, 4) NRS2002, 5) anthropometric measuring, 6) hematological measurement, 7) PG-SGA, 8) body composition, 9) KPS, 10) quality of life assessment (QLQ C30). Duration of hospital stay, the following information will be collected which was nutritional intervention (EN, $\mathrm{PN}$, or $\mathrm{EN}+\mathrm{PN})$, anticancer treatments, length of hospital stay, length of ICU stay, and hospital costs.

\section{Follow-up}

The cohort has been followed after hospital discharge. Vital status will be collected at 30 days, and 1, 2, 3, 4, 5, 7 , and 8 years after recruitment. If the subjects were deceased, date of death and death from cancer or other causes will be identified.

\section{Quality control}

All the information of each recruited patients will be reviewed by a supervisor in each hospital. Data entry in the electronic data capture (EDC) system will be double checked. Databases will be checked by Central Management Team each month, and missing data will be asked to replenish in time. The quality and completeness of the data for each cancer patient are assessed with IARC-crg tools to identify errors, inconsistencies and unusual combinations of cancer site, morphology, sex and/or age at diagnosis.

\section{Data analysis}

Statistical analysis will be performed by SPSS 21.0 software package (Analysis software, Shanghai, co., LTD, 6761805c6989326cbf14). Multiple imputation was conducted to handle missing data.

\section{Nutritional status}

The continuous normal distributed variables will be expressed as means \pm standard deviation (SD) and compared by analysis of variance (ANOVA) or $t$ test. Non-normal distributed continuous variables are represented by median and quartile range and nonparametric tests are used to compare difference between groups. Chi-square test and Wilcoxon Ranksum or Kruskal Wall test are performed for categorical and continuous variables as appropriate. Correlations between independent variables such as nutritional indicators, clinical features, treatment methods and other influencing 
factors and PG-SGA and NRS2002 will be analyzed using logistic regression. Correlation analysis will also be used to determine the association between PG-SGA score and demographic characteristics.

\section{Quality of life}

Spearman tests will be applied to analyze the correlation between nutritional status and QoL scores.

\section{0-days death, hospital costs, hospital stay and influencing factors}

Chi-square test will be used to analyze the influencing factors for 30-day death and logistic multivariate regression model will be used to analyze the independent influencing factors for 30-day death. Hospital stay and hospital costs, are represented by median and quartile range. Comparison of hospital stay and hospital costs between two groups of patients with different nutritional risks and nutritional status will be conducted using MannWhitney test. Logistic regression will be used to analyze the independent influencing factors for hospital stay and hospital costs.

\section{Long time follow-up disease-free survival and overall survival}

Survival rates for 1-8 years will be calculated by Kaplan Meier test and compared using log-rank test; analysis of independent influencing factors related to survival will be performed using multivariate Cox proportional risk regression model.

\section{DISCUSSION}

Patients with cancer are at particularly high risk for malnutrition because both the disease and anticancer treatments. ${ }^{12}$ Nutritional intervention for the management of cancer-related malnutrition will facilitate nutrition support in clinical practice and benefit the clinical outcome. . $^{7,12,25,27}$ The frequent presence of malnutrition in cancer patients can limit their response to even the best therapies if nutritional issues are not appropriately managed ${ }^{30,32}$ Understanding baseline levels of nutritional status, physical function and quality of life is of growing importance in defining individualized supportive care plans for cancer patients. ${ }^{12}$ In this INSCOC study, we have thus far collected more than 30,000 cases from 80 hospitals since 2013 and will recruit additional 20,000 cases in totally 100 hospitals in different regions in China in the next 3 years. The integrity nutritional assessments in INSCOC study have been implemented, including anthropometric measuring, hematological measurement, PG-SGA questionnaire, body composition, physical status, and quality of life. A multi-dimensional assessment of nutritional status at baseline would allow early identification of patients at greatest risk of nutrition status decline and thereby stratification for nutritional interventions in preparation for individualized supportive care plans tolerance and sensitization cancer treatments. Objective, formalized assessment of nutritional status alongside assessment of physical performance and quality of life at baseline may provide additional discriminatory information, which would better inform multidisciplinary team decisions and the preparation for systemic anticancer treatment for these patients.

There are several concerns over the assessment of nutritional status. The exemplary questions are: is there nutritional risk screening in each cancer patient at the first interview with a doctor or investigator? How severe nutritional status should be considered for nutritional intervention or treatment? Is there a risk for overtreatment in malnutritional cancer patients? Does nutritional intervention need to be cancer-type specific? The INSCOC study is a multi-centered, large sample, long time follow-up prospective study. It is hoped that the anticipated results from this study will be able to address the aforementioned concerns. Through this study, we will also validate the PG-SGA questionnaire for Chinese cancer patients. As planned, the validation of the PGSGA will be conducted in 2000 Chinese cancer patients and if possible, we will create a brief Chinese PG-SGA for future studies and if validated, it can be used in future clinical practice.

In summary, this study will aid in identification, prevention, and treatment of reversible elements of malnutrition in cancer patients will help diagnosing malnutrition in cancer patients, sort the related risk factors related the negative outcome, providing a better nutrition evaluation, controlling risk factors, standardizing the nutrition intervention and thus improving clinical outcomes in cancer patients with malnutrition.

Currently, the cohort is being followed with a wellestablished and tested protocol, including record linkage with multiple sources of routinely collected data in China. Through a long follow-up, the INSCOC will provide a valuable opportunity to evaluate many important hypotheses related to cancer and nutritional status that cannot be adequately investigated in studies conducted in Western countries.

\section{ACKNOWLEDGEMENTS}

We thank all the investigators and organizers from different study centers and hospitals for their contributions in various aspects of the present study, including input in study design, review of study protocols, recruitment of the patients, data collection and data sorting, entry and, checking, as well as retrieving miss data. We thank all the participating hospitals for their administrative support for this project. We also thank WINCOME Computer and Technology Corporation for establishing the EDC system for this present study. 
\#Hongxia $\mathrm{Xu}$, Chunhua Song, Chang Wang and Zhenming Fu contributed equally to this work.

Funding: National Science and Technology Major Project from Ministry of Science and Technology of the People's Republic of China, the National Key Research and Development Program to Dr. Hanping Shi (No: 2017YFC1309200)

Conflict of interest: None declared

Ethical approval: The study was approved by the Institutional Ethics Committee

\section{REFERENCES}

1. Siegel RL, Miller KD, Jemal A. Cancer statistics, 2016. CA Cancer J Clin. 2016;66(1):7-30.

2. Chen W, Zheng R, Baade PD, Zhang S, Zeng H, Bray F, et al. Cancer statistics in China, 2015. CA Cancer J Clin. 2016;66(2):115-32.

3. Miller KD, Siegel RL, Lin CC, Mariotto AB, Kramer JL, Rowland JH, et al. Cancer treatment and survivorship statistics, 2016. CA Cancer J Clin. 2016;66(4):271-89.

4. Virizuela JA, Camblor-Álvarez M, Luengo-Pérez LM, Grande E, Álvarez-Hernández J, SendrósMadroño MJ, et al. Nutritional support and parenteral nutrition in cancer patients: an expert consensus report. Clin Transl Oncol. 2018;20(5):619-29.

5. Argilés JM. Cancer-associated malnutrition. Eur J Oncol Nurs. 2005;9(Suppl 2):S39-50.

6. Omlin A, Blum D, Wierecky J, Haile SR, Ottery FD, Strasser F. Nutrition impact symptoms in advanced cancer patients: frequency and specific interventions, a case-control study. J Cachexia Sarcopenia Muscle. 2013;4(1):55-61.

7. Obermair A, Simunovic M, Isenring L, Janda M. Nutrition interventions in patients with gynecological cancers requiring surgery. Gynecol Oncol. 2017;145(1):192-9.

8. Ravasco P, Monteiro-Grillo I, Vidal PM, Camilo ME. Dietary counseling improves patient outcomes: a prospective, randomized, controlled trial in colorectal cancer patients undergoing radiotherapy. J Clin Oncol. 2005;23(7):1431-8.

9. Martin L, Lagergren J, Lindblad M, Rouvelas I, Lagergren P. Malnutrition after oesophageal cancer surgery in Sweden. Br J Surg. 2007;94(12):1496500.

10. Rosania R, Chiapponi C, Malfertheiner P, Venerito M. Nutrition in Patients with Gastric Cancer: An Update. Gastrointest Tumors. 2016;2(4):178-87.

11. Arends J, Bachmann P, Baracos V, Barthelemy N, Bertz H, Bozzetti F, et al. ESPEN guidelines on nutrition in cancer patients. Clin Nutr. 2017;36(1):11-48.

12. Waitzberg DL, Caiaffa WT, Correia MI. Hospital malnutrition: the Brazilian national survey (IBRANUTRI): a study of 4000 patients. Nutrition. 2001;17(7-8):573-80.
13. Ollenschläger G, Viell B, Thomas W, Konkol K, Bürger B. Tumor anorexia: causes, assessment, treatment. Recent Results Cancer Res. 1991;121:249-59.

14. Baldwin C, Weekes CE. Dietary advice for illnessrelated malnutrition in adults. Cochrane Database Syst Rev. 2008;1:CD002008.

15. Rivadeneira DE, Evoy D, Fahey TJ 3rd, Lieberman MD, Daly JM. Nutritional support of the cancer patient. CA Cancer J Clin. 1998;48(2):69-80.

16. Chima CS, Barco K, Dewitt ML, Maeda M, Teran JC, Mullen KD. Relationship of nutritional status to length of stay, hospital costs, and discharge status of patients hospitalized in the medicine service. J Am Diet Assoc. 1997;97(9):975-8.

17. Correia MI, Waitzberg DL. The impact of malnutrition on morbidity, mortality, length of hospital stay and costs evaluated through a multivariate model analysis. Clin Nutr. 2003;22(3):235-9.

18. Pressoir M, Desné S, Berchery D, Rossignol G, Poiree B, Meslier M, et al. Prevalence, risk factors and clinical implications of malnutrition in French Comprehensive Cancer Centres. $\mathrm{Br} \mathrm{J}$ Cancer. 2010;102(6):966-71.

19. Wie GA, Cho YA, Kim SY, Kim SM, Bae JM, Joung H. Prevalence and risk factors of malnutrition among cancer patients according to tumor location and stage in the National Cancer Center in Korea. Nutrition. 2010;26(3):263-8.

20. Sesterhenn AM, Szalay A, Zimmermann AP, Werner JA, Barth PJ, Wiegand S. Significance of autopsy in patients with head and neck cancer. Laryngorhinootologie. 2012;91(6):375-80.

21. Zhang L, Lu Y, Fang Y. Nutritional status and related factors of patients with advanced gastrointestinal cancer. $\mathrm{Br} \quad \mathrm{J}$ Nutr. 2014;111(7):1239-44.

22. Caillet P, Liuu E, Raynaud Simon A, Bonnefoy M, Guerin O, Berrut G, et al. Association between cachexia, chemotherapy and outcomes in older cancer patients: A systematic review. Clin Nutr. 2017;36(6):1473-82.

23. Ottery FD. Rethinking nutritional support of the cancer patient: the new field of nutritional oncology. Semin Oncol. 1994;21(6):770-8.

24. Ravasco P, Monteiro-Grillo I, Camilo M. Individualized nutrition intervention is of major benefit to colorectal cancer patients: long-term follow-up of a randomized controlled trial of nutritional therapy. Am J Clin Nutr. 2012;96(6):1346-53.

25. Temel JS, Greer JA, Muzikansky A, Gallagher ER, Admane S, Jackson VA, et al. Early palliative care for patients with metastatic non-small-cell lung cancer. N Engl J Med. 2010;363(8):733-42.

26. Baldwin C, Spiro A, Ahern R, Emery PW. Oral nutritional interventions in malnourished patients with cancer: a systematic review and meta-analysis. J Natl Cancer Inst. 2012;104(5):371-85. 
27. Barlow R, Price P, Reid TD, Hunt S, Clark GW, Havard TJ, Puntis MC, Lewis WG. Prospective multicentre randomised controlled trial of early enteral nutrition for patients undergoing major upper gastrointestinal surgical resection. Clin Nutr. 2011;30(5):560-6.

28. Wu GH, Liu ZH, Wu ZH, Wu ZG. Perioperative artificial nutrition in malnourished gastrointestinal cancer patients. World J Gastroenterol. 2006;12(15):2441-4.

29. Chi J, Yin S, Zhu Y, Gao F, Song X, Song Z, Lv J, Li M. A Comparison of the Nutritional Risk Screening 2002 Tool With the Subjective Global Assessment Tool to Detect Nutritional Status in Chinese Patients Undergoing Surgery With Gastrointestinal Cancer. Gastroenterol Nurs. 2017;40(1):19-25.

30. Yu K, Zhou XR, He SL. A multicentre study to implement nutritional risk screening and evaluate clinical outcome and quality of life in patients with cancer. Eur J Clin Nutr. 2013;67(7):732-7.

31. Zhang L, Wang C, Sha SY, Kwauk S, Miller AR, Xie MS, et al. Mini-nutrition assessment, malnutrition, and postoperative complications in elderly Chinese patients with lung cancer. J BUON. 2012;17(2):323-6.

32. Wu B, Yin TT, Cao W, Gu ZD, Wang X, Yan M, et al. Validation of the Chinese version of the Subjective Global Assessment scale of nutritional status in a sample of patients with gastrointestinal cancer. Int J Nurs Stud. 2010;47(3):323-31.

33. Cong M, Wang J, Fang Y, Liu Y, Sun M, Wu Q, et al. A multi-center survey on dietary knowledge and behavior among inpatients in oncology department. Support Care Cancer. 2018;26(7):2285-92.
34. Wu BW, Yin T, Cao WX, Gu ZD, Wang XJ, Yan $\mathrm{M}$, et al. Clinical application of subjective global assessment in Chinese patients with gastrointestinal cancer. World J Gastroenterol. 2009;15(28):3542-9.

35. Kondrup J, Rasmussen HH, Hamberg O, Stanga Z, Ad Hoc ESPEN Working Group. Nutritional risk screening (NRS 2002): a new method based on an analysis of controlled clinical trials. Clin Nutr. 2003;22(3):321-36.

36. Aaronson NK, Ahmedzai S, Bergman B, Bullinger M, Cull A, Duez NJ, et al. The European Organization for Research and Treatment of Cancer QLQ-C30: a quality-of-life instrument for use in international clinical trials in oncology. J Natl Cancer Inst. 1993;85(5):365-76.

37. Fayers PMANK, Bjorda IKN, JLKDJAOI. On behalf of the EORTC Quality of Life Group. The EORTC QLQ-C30 Scoring Manual (3rd Edition). 2001.

38. Lochs H, Allison SP, Meier R, Pirlich M, Kondrup $\mathrm{J}$, Schneider S, et al. Introductory to the ESPEN Guidelines on Enteral Nutrition: Terminology, definitions and general topics. Clin Nutr. 2006;25(2):180-6.

39. Buzby GP, Knox LS, Crosby LO, Eisenberg JM, Haakenson CM, McNeal GE, et al. Study protocol: a randomized clinical trial of total parenteral nutrition in malnourished surgical patients. Am J Clin Nutr. 1988;47(2 Suppl):366-81.

Cite this article as: $\mathrm{Xu} \mathrm{H}$, Song $\mathrm{C}$, Wang C, Fu Z, Guo Z, Shi H, et al. Investigation on nutrition status and clinical outcome of patients with common cancers in Chinese patients: a multicenter prospective study protocol. Int J Clin Trials 2020;7(2):94-102. 